\section{Approach Maneuvers for Autonomous Landing Using Visual Servo Control}

\author{
FLORENT LE BRAS \\ Direction Générale de l'Armement \\ TAREK HAMEL \\ University of Nice Sophia Antipolis \\ ROBERT MAHONY \\ Australian National University \\ CHRISTIAN BARAT \\ University of Nice Sophia Antipolis \\ JULIEN THADASACK \\ Direction Générale de l'Armement
}

\begin{abstract}
A nonlinear image-based visual servo control algorithm for autonomous landing of a fixed wing aircraft is described. The primary sensor system is a vision sensor yielding a sequence of images from which $2 \mathrm{D}$ linear and point features of the runway are extracted. The first two phases of a landing maneuver, alignment to the runway and glide (descent to the runway) are treated in the work presented here. The final landing maneuvers, flare to touchdown, and taxiing require additional sensor modalities and are not treated. The proposed control scheme deals with unknown wind conditions and incorporates the full nonlinear dynamics of the airplane. Simulation results based on realistic environmental conditions and measurement noise are presented that validate the control design approach.
\end{abstract}

Manuscript received December 31, 2011; revised October 1, 2012 released for publication April 3, 2013.

DOI. No. 10.1109/TAES.2013.110780

Refereeing of this contribution was handled by I. Hwang.

This research was partly supported by the Australian Research Council through the Fellowship FT0991771 "Foundations of Vision Based Control of Robotic Vehicles".

Authors' addresses: F. Le Bras, French Direction Générale de l'Armement (Technical Directorate), Bagneux, France; T. Hamel and C. Barat, UNSA-I3S-CNRS, Nice-Sophia Antipolis, France. E-mail: (thamel@i3s.unice.fr). R. Mahoney, Research School of Engineering, ANU, Canberra, Australia; J. Thadasack, French Direction Générale de l'Armement (Technical Directorate), Bruz, France.

0018-9251/14/\$26.00 @ 2014 IEEE

\section{INTRODUCTION}

In the past decade $50 \%$ of airplanes accidents (responsible for $40 \%$ of fatalities) have occurred during the approach and landing phases of flight [1]. The main reason for these accidents is pilot error, generally exacerbated by difficult weather conditions including strong transverse wind, rain, or fog [1]. The development of reliable autonomous landing systems has been an important research area for some time [2, 3], receiving additional emphasis recently with the emergence of unmanned aerial vehicle (UAV) systems. The key requirement for an effective autonomous landing system is a robust and reliable estimate of the position of the aircraft with respect to the runway during the approach phase. Most major passenger airports are now equipped with an instrument landing system (ILS) or differential GPS to provide a primary measurement system for autonomous control of landing. Such systems are expensive, require constant maintenance, and may require a large-scale accurate survey of the airport, making them unsuitable for the large number of smaller recreational and small-scale commercial airports in the world. The operational deployment of UAV systems also naturally leads to unstructured and noninstrumented runways, or to exposure to threats like electromagnetic interference that potentially impact GPS reliability or compromise the data link associated with an ILS. Vision imaging systems are one of the most promising technologies that provide a robust, secure sensor modality for regulating the landing manoeuvre of an aircraft or UAV on a noninstrumented and unsurveyed runway. Cameras are relatively low cost, can be easily embedded in a light aircraft or UAV system, and image processing techniques have developed a level of maturity consistent with the required accuracy for landing [4]. Existing image-based landing algorithms use a position-based visual servo control framework [5] where the vision system is used to estimate the aircraft position with respect to the runway and this information is used to replace the ILS input in a classical control strategy $[2,3]$. Such an approach still requires an accurate surveyed model of at least the runway, if not the airport surrounds, and careful calibration of the intrinsic and extrinsic parameters of the camera, making it unsuitable as a general landing aiding system in a recreational aircraft or UAV system. Image-based visual servo techniques offer the possibility to overcome these issues [6] and several initial results for image-based visual servo control of landing manoeuvres have already been obtained [7-9].

In this paper we provide a robust and effective image-based visual servo control algorithm to regulate the approach phase of landing (alignment and glide). The proposed approach requires no external instrumentation, no a priori knowledge of the airport and is robust to significant crosswinds. The visual information extracted from the image sequence is the edges of the runway borders, represented as Plücker coordinates [10] (for regulation of alignment) and the runway front corner 
points (for regulation of descent). The algorithm incorporates a robust adaptive estimate of crosswind and corrects for these effects in the closed-loop system response, leading to considerable performance and robustness advantages compared with preexisting works [7-9]. A key contribution of the paper is the incorporation of a saturation on the crosswind estimate, improving the response of the closed-loop system, avoiding integrator wind-up effects if there is dropped data, and improving the basin of attraction of the closed-loop system. In addition, the proposed algorithm takes explicit account of the aircraft flight dynamics, an advance on prior work. A comprehensive set of realistic simulations have been undertaken to verify the performance of the proposed algorithm based on the visual output from a flight simulator system for an established realistic model of real world aircraft dynamics.

The paper comprises six sections including the Introduction. Section II presents the aircraft flight dynamics model used. Section III presents a classical control architecture that stabilizes a part of the flight dynamics of the vehicle and leaves free the degrees of motion that will be regulated by the visual servo control. The main contribution of the paper is given in Section IV. First we present the image features used to control the aircraft while landing, present the proposed control design and go on to analyse its stability and performance properties. Finally, in Section V a simulation study illustrates the performance of the proposed controller using a realistic model of an embedded camera and realistic flight dynamics. Section VI provides a short summary of conclusions.

\section{AIRCRAFT MODELING}

In this paper we focus on aircraft that can be modeled as a rigid body associated with the airframe subjected to aerodynamic forces. The dynamics of the aircraft are modeled by Newton's equations with external forces and moments due to gravity, lift, and drag and the forces and torques applied by control inputs.

Let $I=\left(E_{x}, E_{y}, E_{z}\right)$ denote an inertial frame of reference. The axis $E_{z}$ is oriented toward the center of the Earth, while $E_{x}$ is oriented along the runway and $E_{y}$ is chosen to make a right-hand frame. Let $B=\left(e_{x}, e_{y}, e_{z}\right)$ denote the body-fixed frame attached to the airframe with $e_{x}$ towards the nose of fuselage, $e_{z}$ down, and $e_{y}$ along the wings. The orientation of the aircraft is given by a rotation $R: B \rightarrow I$ and parameterized using the classical 'roll', 'pitch' and 'yaw' Euler angles ( $\phi$ (roll), $\theta$ (pitch), $\psi$ (yaw)) commonly employed in aerodynamic applications [11, pg. 608].

The flow frame (also termed the wind or airspeed frame), denoted $F=\left(e_{x}^{f}, e_{y}^{f}, e_{z}^{f}\right)$, is oriented with $e_{x}^{f}$ in the direction of the perceived wind on the vehicle, while $e_{z}^{f}$ is chosen to lie in the plane of symmetry of the vehicle orthogonal to $e_{y}$, and $e_{y}^{f}$ makes up the right-hand frame. By construction the velocity of the vehicle in the flow

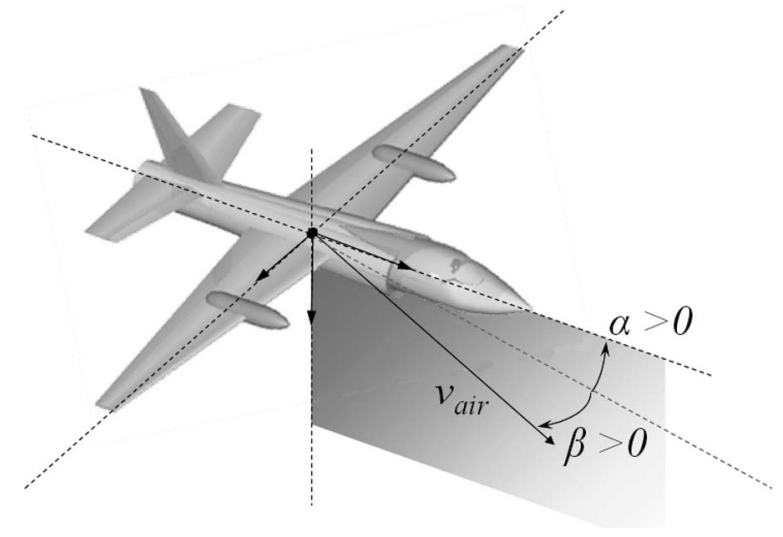

Fig. 1. Representation of angle-of-attack, sideslip angle and airspeed. Here $v_{\text {air }}$ is denoted $v_{a}$ in the text to save space.

frame always lies in direction $e_{x}^{f}$. In the body-fixed frame $B$, however, the velocity of the vehicle with respect to the wind, expressed in the body-fixed frame is

$$
v_{a}=v-v_{w} \in B
$$

where $v_{w}$ denotes the wind velocity and $v$ is the vehicle velocity, both measured with respect to the inertial frame and expressed in the body-fixed frame. Note that $v_{a}$ is not, in general, parallel to $e_{x}$.

We define an orientation matrix $R_{\alpha, \beta}: F \rightarrow B$ to describe the orientation of the airflow frame in terms of the angle-of-attack $\alpha$, and the side-slip angle $\beta$, [12] (see Fig. 1)

$$
R_{\alpha, \beta}=\left(\begin{array}{ccc}
c_{\alpha} c_{\beta} & c_{\alpha} s_{\beta} & -s_{\alpha} \\
s_{\beta} & c_{\beta} & 0 \\
s_{\alpha} c_{\beta} & -s_{\alpha} s_{\beta} & c_{\alpha}
\end{array}\right)
$$

where $c_{(.)}$and $s_{(.)}$are shorthand abbreviations of $\cos ($.$) and$ $\sin ($.$) respectively. A less common orientation matrix$ $R_{\mu, \gamma, \chi}: F \rightarrow I$ can be used to define the orientation of the airspeed frame with respect to the inertial frame in terms of the aerodynamic bank angle $\mu$, the flight path angle $\gamma$, and the heading angle $\chi$

$$
R_{\mu, \gamma, \chi}=\left(\begin{array}{ccc}
c_{\gamma} c_{\chi} & s_{\gamma} s_{\mu} c_{\chi}-c_{\mu} s_{\chi} & s_{\gamma} c_{\mu} c_{\chi}+s_{\mu} s_{\chi} \\
c_{\gamma} s_{\chi} & s_{\gamma} s_{\mu} s_{\chi}+c_{\mu} c_{\chi} & s_{\gamma} c_{\mu} s_{\chi}-s_{\mu} c_{\chi} \\
-s_{\gamma} & c_{\gamma} s_{\mu} & c_{\gamma} c_{\mu}
\end{array}\right)
$$

Consider the situation where the aircraft is in normal flight, that is cruising at subsonic velocity while avoiding stall phenomenon. In such a scenario it is usual to assume incompressible flow leading to linear variation of the aerodynamic forces with dynamic pressure [12]

$$
\bar{Q}=\frac{1}{2} \rho V_{a}^{2}
$$

where $\rho$ is the air density and $V_{a}$ represents the norm of the airspeed $v_{a}$. The translational aerodynamic forces can be expressed in the wind frame by dimensionless 
coefficients [12]

$$
\left(F_{a}^{x}, F_{a}^{y} F_{a}^{z}\right)^{\top}=\bar{Q} S\left(-C_{x}, C_{y},-C_{z}\right)^{\top}
$$

where $S$ represents the aircraft reference surface (normally the wings surface). To first-order approximation, the coefficients satisfy

$$
C_{y}=C_{y, \beta} \beta, C_{z}=C_{z, \alpha} \alpha, C_{x}=C_{x_{0}}
$$

The lift $C_{z}$ can be modeled by a lift coefficient $C_{z, \alpha} \alpha$ acting on the angle-of-attack $\alpha$. A common notation is to introduce a constant offset in the angle of attack $\alpha_{0}$, that can be thought of as the effective angle-of-attack of the lift surface when $\alpha$ is nominally zero. This leads to a lift coefficient $C_{z}=C_{z, \alpha}\left(\alpha+\alpha_{0}\right)$. The offset $\alpha_{0}$ comes from the fact that most wing profiles will generate lift even when the angle-of-attack is zero, not to mention that most aircraft are constructed with the wing at a slight angle to the main fuselage. It is equally straightforward to define the body-fixed frame suitably such that $\alpha_{0}=0$. Since this simplifies the algebra in the derivations undertaken in the sequel we take this approach.

The aerodynamic moments (expressed in the body-fixed frame) are characterized by dimensionless coefficients [12]:

$$
\begin{aligned}
\left(\begin{array}{l}
\Gamma_{a}^{x} \\
\Gamma_{a}^{y} \\
\Gamma_{a}^{z}
\end{array}\right) & =\bar{Q} S l\left(\begin{array}{l}
C_{l} \\
C_{m} \\
C_{n}
\end{array}\right) \\
& =\bar{Q} S l\left(\begin{array}{l}
C_{l, x} \frac{l \Omega_{x}}{2 V_{a}}+C_{l, z} \frac{l \Omega_{z}}{2 V_{a}}+C_{l, \delta} \delta_{l} \\
C_{m 0}+C_{m, \alpha} \alpha+C_{m, y} \frac{l \Omega_{y}}{2 V_{a}}+C_{m, \delta} \delta_{m} \\
C_{n, \beta} \beta+C_{n, z} \frac{l \Omega_{z}}{2 V_{a}}+C_{n, \delta} \delta_{n}
\end{array}\right)
\end{aligned}
$$

where $\left(\Omega_{x}, \Omega_{y}, \Omega_{z}\right)$ are the components of the orientation velocity $\Omega$ and represent the pitch, roll, and yaw rates of the body-fixed frame, respectively; $l$ is the aircraft reference length, typically the wing span, and $\left(\delta_{l}, \delta_{m}, \delta_{n}\right)$ are deflections of the airplane control surfaces (ailerons, elevator, and rudder).

We assume that the wind speed is slowly time varying in the inertial frame with respect to the aircraft dynamics, that is $\frac{d}{d t}\left(R v_{w}\right) \simeq 0$. The standard dynamic model for fixed-wing aircraft is given by [12]

$$
\begin{gathered}
\dot{\xi}=R\left(v_{a}+v_{w}\right) \\
\dot{v}_{a}=-\Omega \times v_{a}+\mathrm{g} E_{z}+\frac{T}{m} e_{x} \\
+\frac{\bar{Q}}{m} S\left(-C_{x} e_{x}^{f}+C_{y} e_{y}^{f}-C_{z} e_{z}^{f}\right) \\
\dot{R}=R \Omega_{\times} \\
\mathrm{I} \dot{\Omega}=-\Omega \times \mathrm{I} \Omega+\bar{Q} \operatorname{Sl}\left(C_{l} e_{x}+C_{m} e_{y}+C_{n} e_{z}\right)
\end{gathered}
$$

where $\mathrm{g} E_{z}$ is the gravitational acceleration. The notation $\Omega_{\times}$denotes the skew-symmetric matrix such that
$\Omega_{\times} y=\Omega \times y$ for the vector cross-product $\times$ and any vector $y \in \mathbb{R}^{3}$. In normal flight the lift compensates the gravitational force. Lateral acceleration is obtained by tilting the lift component, a flight strategy termed bank-to-turn in the aerospace literature [13]. The lift magnitude and the orientation dynamics are governed by means of the control surfaces deflection $\left(\delta_{l}, \delta_{m}, \delta_{n}\right)$. The longitudinal dynamics are governed by means of the propulsion system, modeled by the axial force $T e_{x}(8)$, which compensates for the drag and ensures regulation of airspeed around the desired set point.

Only partial measurements of state variables of dynamics (8) are available. The inertial measurement unit along with suitable filtering algorithms provides the angular velocity $\Omega$ and an estimate of orientation matrix $R$. A set of Pitot tubes provides the measurement of the airspeed $v_{a}$ in magnitude and direction $\left(V_{a}, \alpha, \beta\right)$ and the dynamic pressure $\bar{Q}$ can be calculated using (4).

Combining the measurement of the airspeed direction with the measurement of $R$, we get measurements of $(\mu, \gamma, \chi)$. The measurement of the position of the aircraft $\xi$ (with respect to the inertial frame fixed to the runway) and the wind velocity $v_{w}$ are not available.

\section{FLIGHT CONTROL}

In this section we propose the control architecture that we exploit for the image-based visual servo control problem. The architecture is based on the classical division of aircraft control into navigation (choice of trajectory, i.e. the landing trajectory described in Section IV for our application), guidance (also termed outer-loop, is the control of the trajectory of the vehicle, ensured here by the proposed IBVS control scheme) and flight control (consisting of two inner-loops: one to perform the regulation of the airspeed magnitude and the other to perform the regulation of the attitude of the vehicle). The control architecture relies on a time scale separation between the guidance (slow dynamics) and the flight control more reactive (fast dynamics).

To properly pose the control task, we first describe the model used for the guidance control problem and then we define the set point for the flight control algorithm.

The outer-loop control design or equivalently the guidance control loop considered in the sequel is based on a dynamic reduction of the full aircraft dynamics (8). More precisely the dynamics that we consider for the guidance are given by

$$
\begin{gathered}
\dot{\xi}=R\left(v_{a}+v_{w}\right) \\
\dot{v}_{a}=-\Omega \times v_{a}+F_{c} \\
\dot{v}_{w}=-\Omega \times v_{w}
\end{gathered}
$$

where the unknown constant wind speed $v_{w} \in B$ has dynamics induced by the ego-motion of the body-fixed-frame representation. The vectorial term $F_{c}$ gathers together the external forces of (8b). It is used as the control input for the guidance control problem involving 
the dynamics (9). The value of $F_{c}$ that results from the guidance control design is used as the reference set point for a high gain flight control. The magnitude of $F_{c}$ is used as the set point for the airspeed regulation control while it's orientation is used as reference for attitude regulation.

Due to the complexity and trade-offs implicit in the nature of the aerodynamic lift and thrust equations discussed in Section II, the use of (9b) without any other constraint does not ensure the well-posedness of the inner-loop control problem. To overcome this issue we impose two additional constraints on the vehicle's motion.

1) Constant airspeed: that is the norm $V_{a}=\left|v_{a}\right|$ is held constant.

2) Bank-to-turn: that is the vehicle will bank into any turn to ensure that the side-slip angle is zero $\beta=0$ while keeping $V_{a}$ constant.

The first task we consider is to provide a separate control loop to regulate the airspeed of the vehicle.

Recalling that $V_{a}=\left|v_{a}\right|$, one has

$$
\begin{aligned}
\dot{V}_{a} & =\frac{v_{a}^{\top} \dot{v}_{a}}{V_{a}} \\
& =\frac{v_{a}}{V_{a}}\left(\mathrm{~g} E_{z}+\frac{T}{m} e_{x}-\frac{\bar{Q}}{m} S\left(C_{x} e_{x}^{f}-C_{y} e_{y}^{f}+C_{z} e_{z}^{f}\right)\right) .
\end{aligned}
$$

Using the fact that $\frac{v_{a}}{V_{a}}=e_{x}^{f}$ and recalling (2) and (3), it follows that

$$
\dot{V}_{a}=-\frac{\bar{Q}}{m} S C_{x}+\frac{T}{m} \cos \alpha \cos \beta-\mathrm{g} \sin \gamma
$$

with $\sin \gamma=\left\langle e_{x}^{f}, E_{z}\right\rangle$ and $\cos \alpha \cos \beta=\left\langle e_{x}^{f}, e_{x}\right\rangle$. Assume $\beta \approx 0$ and define the control thrust according to a PI-type control action

$$
\begin{aligned}
T & =\frac{T_{\gamma}^{\star}}{\cos \alpha}, \quad \text { with } \\
T_{\gamma}^{\star} & =\bar{Q} S C_{x}+m \mathrm{~g} s_{\gamma}-k_{V, p}\left(V_{a}-V_{a}^{\star}\right)-k_{V, I} \int\left(V_{a}-V_{a}^{\star}\right) d t
\end{aligned}
$$

where $\left(k_{V, p}, k_{V, I}\right)$ are two strictly positive control gains and the intermediary variable $T_{\gamma}^{\star}:=T_{\gamma}^{\star}\left(V_{a}, \gamma\right)$ is independent of $\alpha$. Define

$$
L_{v}=\tilde{V}_{a}^{2}+\frac{k_{V, I}}{m}\left(\int \tilde{V}_{a} d t\right)^{2}
$$

where $\tilde{V}_{a}=V_{a}-V_{a}^{\star}$. Differentiating $L_{v}$ yields

$$
\dot{L}_{v}=-\frac{2}{m} k_{V, p} \tilde{V}_{a}^{2} \text {. }
$$

It follows that the norm of airspeed is locally exponentially stable under the proposed control. ${ }^{1}$ The remainder of the section considers the design of the flight

\footnotetext{
${ }^{1}$ Note that statements about global exponential stability for a system model that is based on a linearisation argument are pointless. The system model is only valid for small angle-of-attack and side-slip angles.
}

control algorithm to ensure that an arbitrary $F_{c}$ is obtained in (9b). Recalling (8) and assuming that $V_{a}$ is regulated to a constant desired value $V_{a}^{\star}$, it follows that

$$
\dot{v}_{a}=-\Omega \times v_{a}+\pi_{v_{a}} \frac{1}{m} F_{\text {ext }}
$$

where $\pi_{v_{a}}$ denotes the projection onto the plane orthogonal to $v_{a}$,

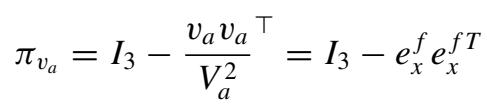

with $I_{3}$ the $3 \times 3$ identity matrix. Recalling (8b) one has

$$
F_{e x t}=\left(m \mathrm{~g} R^{\top} E_{z}+T e_{x}+\bar{Q} S\left(-C_{x} e_{x}^{f}+C_{y} e_{y}^{f}-C_{z} e_{z}^{f}\right)\right) \text {. }
$$

From $(9 b)$ it follows that

$$
F_{c}=\pi_{v_{a}} \frac{1}{m} F_{\text {ext }} .
$$

The goal is now to express this equation in a suitable form to generate the set point for the flight control inner-loop dedicated to the attitude.

Applying the constraint on the sideslip angle $\beta \equiv 0$ will derive conditions on the angle-of-attack $\alpha$ and bank-angle $\mu$ that assign the desired value of $F_{c}$. Note that the flight path angle $\gamma$ and the heading angle $\chi$

parameterize the direction $v_{a} /\left|v_{a}\right|$ of motion of the vehicle and must be treated as free variables in the design of the attitude flight control algorithm. Expanding $F_{c}$ in the flow frame of reference one obtains

$$
F_{c}=\left\langle\frac{1}{m} F_{\text {ext }}, e_{y}^{f}\right\rangle e_{y}^{f}+\left\langle\frac{1}{m} F_{\text {ext }}, e_{z}^{f}\right\rangle e_{z}^{f}
$$

since the component in the direction $e_{x}^{f}$ is zero. Consider just the $e_{z}^{f}$ component firstly and substituting for (14), it yields

$$
\left\langle\frac{1}{m} F_{\text {ext }}, e_{z}^{f}\right\rangle=\left\langle\mathrm{g} R^{\top} E_{z}+\frac{T}{m} e_{x}, e_{z}^{f}\right\rangle-\frac{\bar{Q}}{m} S C_{z} .
$$

Recall that $\left\langle e_{x}, e_{z}^{f}\right\rangle=-\sin \alpha(2)$ and $\left\langle E_{z}, e_{z}^{f}\right\rangle=$ $\cos \gamma \cos \mu$ (3). With $\beta=0$, then (6) along with (12), imply that

$$
\left\langle F_{c}, e_{z}^{f}\right\rangle=\left(-\frac{\bar{Q} S}{m} C_{z, \alpha} \alpha+\mathrm{g} \cos \gamma \cos \mu-\tan \alpha \frac{T_{\gamma}^{\star}}{m}\right) .
$$

Similarly, with $\beta=0$ the component $C_{y}$ of (14) is equal to zero. From (2) and (3) one has $\left\langle e_{x}, e_{y}^{f}\right\rangle=\cos \alpha \sin \beta=0$ and $\left\langle E_{z}, e_{y}^{f}\right\rangle=\cos \gamma \sin \mu$, respectively. Hence, the component of $F_{c}$ in the direction $e_{y}^{f}$ is given by

$$
\left\langle F_{c}, e_{y}^{f}\right\rangle=\mathrm{g} \cos \gamma \sin \mu .
$$

Finally, one can write

$$
F_{c}(\alpha, \mu)=\left(-\frac{\bar{Q} S}{m} C_{z, \alpha} \alpha+\mathrm{g} c_{\gamma} c_{\mu}-\tan \alpha \frac{T_{\gamma}^{\star}}{m}\right) e_{z}^{f}+\mathrm{g} c_{\gamma} s_{\mu} e_{y}^{f}
$$

where the right-hand side depends only on $\alpha$, and $\mu$ and other known terms $\left(\gamma, \bar{Q}, T_{\gamma}^{\star}\right)$. 
Note that the relationship between $F_{c}$ and $(\alpha, \mu)$ defined by (15) is at least locally one-to-one.

Consequently, this relationship can be used to define reference signals $\left(\alpha^{\star}, \mu^{\star}\right)$ once the desired value $F_{c}^{\star}$ of the vectorial term $F_{c}$ is assigned. The desired bank angle $\mu^{\star}$ is isolated as a function of $F_{c}^{\star}$ by taking the $e_{y}^{f}$ component of (15)

$$
\mu^{\star}=\arcsin \frac{\left\langle F_{c}^{\star}, e_{y}^{f}\right\rangle}{\mathrm{g} \cos \gamma} .
$$

It is, however, more complex to derive a closed-form expression for the desired angle-of-attack. Substituting for $\mu^{\star}$ and taking the $e_{z}^{f}$ component of (15) yields

$$
\left\langle F_{c}^{\star}, e_{z}^{f}\right\rangle=-\frac{\bar{Q} S}{m} C_{z, \alpha} \alpha^{\star}+\mathrm{g} \cos \gamma \cos \mu^{\star}-\tan \alpha^{\star} \frac{T_{\gamma}^{\star}}{m}
$$

and this nonlinear algebraic relationship can be solved numerically. Alternatively, computing the linearisation of the term $\tan \alpha^{\star}$ in (17), replacing $\mu^{\star}$ by (16), and solving explicitly yields

$$
\alpha^{\star}=m \frac{\sqrt{\mathrm{g}^{2} \cos ^{2} \gamma-\left\langle F_{c}^{\star}, e_{y}^{f}\right\rangle^{2}}-\left\langle e_{z}^{f}, F_{c}^{\star}\right\rangle}{T_{\gamma}^{\star}+\bar{Q} S C_{z, \alpha}} .
$$

Since the expression of $F_{c}^{\star}$ is the subject of the next section, it remains to describe the "high gain" inner-loop flight controller that stabilizes the orientation dynamics by regulating $(\beta, \alpha, \mu)$ to the reference $\left(0, \alpha^{\star}, \mu^{\star}\right)$ defined above. We first consider the dynamics of $(\beta, \alpha, \mu)$ along with the orientation velocity dynamics of the aircraft.

$$
\begin{aligned}
\dot{\mu}=\Omega_{x, a}+\left(\Omega_{y, a} \sin \mu+\Omega_{z, a} \cos \mu\right) \tan \gamma \\
\dot{\alpha}=\Omega_{y}-\left(\Omega_{x} \cos \alpha+\Omega_{z} \sin \alpha\right) \tan \beta \\
+\frac{1}{m V_{a} \cos \beta}\left(\bar{Q} S C_{z}-m \mathrm{~g} \cos \gamma \cos \mu+\cos \beta \sin \alpha T\right)
\end{aligned}
$$

$$
\begin{aligned}
& \dot{\beta}= \Omega_{x} \sin \alpha-\Omega_{z} \cos \beta \cos \alpha+\frac{1}{m V_{a}}\left(-\bar{Q} S C_{y}\right. \\
&+m \mathrm{~g} \cos \gamma \sin \mu-\sin \beta T) \\
& \mathrm{I} \dot{\Omega}=-\operatorname{sk}(\Omega) \mathrm{I} \Omega+\bar{Q} \operatorname{sl}\left(C_{l}, C_{m}, C_{n}\right)^{\top}
\end{aligned}
$$

where

$$
\begin{gathered}
\Omega_{x, a}=\left(\Omega_{x} \cos \alpha+\Omega_{z} \sin \alpha\right) \cos \beta+\left(\Omega_{y}-\dot{\alpha}\right) \sin \beta \\
\Omega_{y, a}=\left(\Omega_{y}-\dot{\alpha}\right) \cos \beta-\left(\Omega_{x} \cos \alpha+\Omega_{z} \sin \alpha\right) \sin \beta \\
\Omega_{z, a}=\dot{\beta}-\Omega_{x} \sin \alpha+\Omega_{z} \cos \alpha .
\end{gathered}
$$

Now define

$$
\eta_{1}:=\left(\mu, \alpha, \beta, \frac{l \Omega^{\top}}{V_{a}}\right)^{\top} \text {, and } \eta_{0}:=\left(V_{a}, \alpha^{*}, \beta^{*}, \gamma\right)^{\top} \text {. }
$$

The resulting dynamics of $\eta_{1}$ (19) long with (20)) are complex due to the various coupling effects and the strong

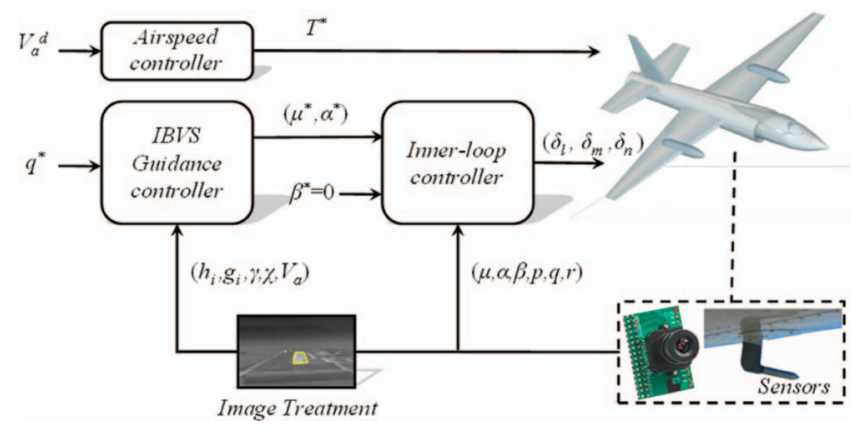

Fig. 2. Proposed control architecture for a fixed-wing aircraft.

nonlinearities. Therefore, seeking a good control-oriented model and designing appropriate controller for these dynamics is one of the challenging tasks in developing aircraft technologies. The high complexity of the dynamics can be tackled using the LPV (linear parameter varying) control approach. The first step in such an approach is to generate an LPV model from (19) along with (20). Several approaches can be used to obtain reliable LPV models, including Jacobian linearisation and state transformation [14]. The approach taken here is that described in [15]. We assume that $V_{a}$ is constant and $\left(\alpha^{*}, \mu^{*}\right)$ along with $\gamma$ are slowly time varying with respect to orientation dynamics. The system dynamics dynamics are linearised about the desired equilibrium induced by $(\beta, \alpha, \mu)=\left(0, \alpha^{\star}, \mu^{\star}\right)$. The key advantage of the LPV design paradigm, lies in the fact that it associates a linear system with each operating point of the attitude dynamics analysis, not just the equilibrium operating points. The resulting dynamics are

$$
\dot{\tilde{\eta}}_{1}=A\left(\eta_{0}\right) \tilde{\eta}_{1}+D\left(\eta_{0}\right)+B\left(\eta_{0}\right) \delta
$$

where $\tilde{\eta}_{1}=\left(\eta_{1}-\eta_{1}^{\star}\right)$ along with $\eta_{1}^{\star}=\left(\mu^{\star}\left(\eta_{0}\right), \alpha^{\star}\left(\eta_{0}\right), 0\right.$, $\left.\frac{1}{V_{a}}\left(e_{x}^{f} \times F_{c}\left(\eta_{0}\right)\right)^{\top}\right)^{\top}$ and finally $\delta=\left(\delta_{l}, \delta_{m}, \delta_{n}\right)^{\top}$ denotes the control surface inputs governing the aerodynamic moments (7). The explicit expressions for the matrices ( $A$, $B, D)$ are given in the Appendix. The full details of this control design are given in [15] and are beyond the scope of the present paper. The control obtained in [15] ensures local exponential stability of $(\beta, \alpha, \mu)$ to the reference set point $\left(0, \alpha^{\star}, \mu^{\star}\right)$ with the required high gain.

Finally, to conclude this section, Fig. 2. provides a block diagram representation of the overall control architecture.

\section{IMAGE-BASED VISUAL SERVO CONTROL FOR THE APPROACH MANEUVERS OF AUTONOMOUS LANDING}

This section describes a guidance control law to perform autonomous landing of the airplane using a vision-based controller. The section begins with a discussion of the landing phases, before going on to the development of the image-based cues considered, and then developing the guidance control laws consisting of assigning the desired value $F_{c}^{\star}$ of $F_{c}$. 


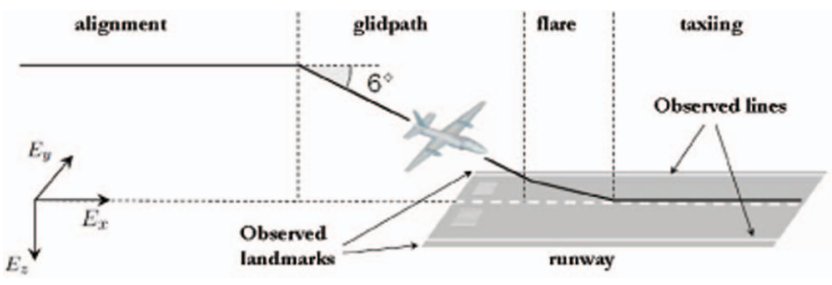

Fig. 3. Representation of the different phases of a landing maneuver.

Landing maneuver is the most demanding phase of a flight due to the precision required and the increased workload on the pilot. A landing maneuver is composed of four phases (see Fig. 3).

1) Alignment: the aircraft aligns with the runway at a fixed desired altitude from the ground. For this maneuver, the border-lines of the runway are used as the primary pose information.

2) Glide approach: the airplane tracks a straight-line descending path, while keeping the alignment with respect to the runway axis. For this maneuver the image features are the coordinates of some specific marks on the runway and the runway axis.

3) Flare to touch down: This maneuver is triggered when the vehicle reaches the runway. First the vehicle ceases to descend and tracks along the center line of the runway at a set height (around $20 \mathrm{~m}$ for large jet vehicles). The vehicle then initiates a flare manoeuvre that reduces airspeed to just above stall speed while slowly descending until the landing carriage touches. The two phases of this maneuver are usually undertaken almost simultaneously by a pilot.

4) Taxiing: The last phase of landing begins when the airplane tires touch the runway. The airplane is then rolling while reducing its velocity to zero.

In this section we focus on the two first maneuvers with some consideration of the first phase of the third maneuver. For full-sized airplanes a full flare to touch down manoeuvre involves regulation of the vertical speed of the aircraft and requires additional sensor modality such as the measurement of optical flow [16]. The taxiing phase is a postflight maneuver which is described by different dynamics and requires a different control strategy [17].

The visual features considered are the images of a collection of $n(n \geq 2)$ parallel lines (i.e., the edges of, or painted lines on, the runway, etc.) along with some specific marks on the runway (i.e., corners of the runway, or a painted threshold target, etc.). The unit direction vector of the lines is denoted $\mathbf{u}$ in the inertial frame and $U$ in the body-fixed frame. We assume that camera-fixed frame coincides with the body-fixed frame and that the image features remain in the camera field of view throughout the flight, a reasonable assumption given the nature of the maneuver considered.

Two different position-like error terms are derived from the visual features. The first is based on the bi-normalized Plücker coordinates of the observed parallel

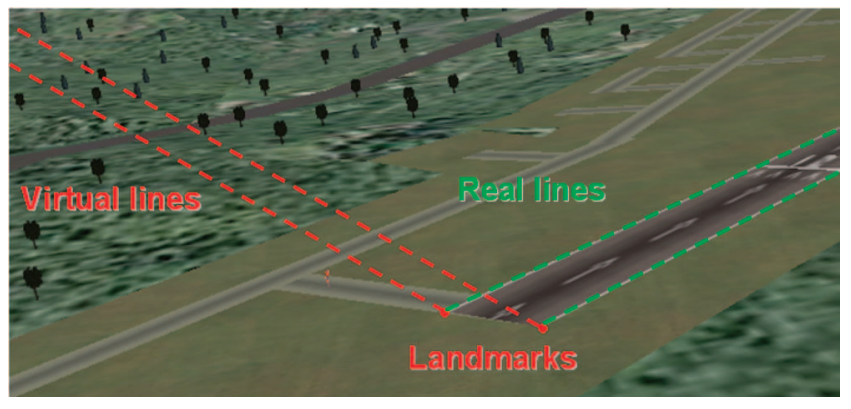

Fig. 4. Representation of the (real) lines used for alignment phases (simply given by the runway's edges) and of the virtual lines used for the descent maneuver (in red), these lines pass through the runway's corners.

lines and is used to perform the alignment phase of the control algorithm. The second is based on the measurement of two landmarks on the runway, and is used to regulate the glide approach During the glide approach the bi-normalized Plücker coordinates of two virtual lines, derived from the observed landmarks, are used to maintain alignment.

The two border lines of the runway form a natural parallel pair of lines in the world frame. An explicit representation of lines in Euclidean space, based on the bi-normalized Plücker coordinates representation, is used in this paper [10] (see Fig. 5). The bi-normalized Plücker coordinates of the $i$-th line, expressed in the body-fixed frame, are given by two unit vectors $\left(h_{i}, U\right)$ such that

$$
h_{i}=\frac{X_{i} \times U}{\left\|X_{i} \times U\right\|} \in \mathcal{B}, U \in \mathcal{B}
$$

where $\boldsymbol{X}_{i}$ denotes a vector between the camera and any point on the considered line. The image of the same line on the image plane is also a line. The bi-normalized Plücker coordinates of this line are denoted $\left(h_{i}, u_{i}\right)$ in $\mathcal{B}$ where the first coordinate is the same as for the physical runway line due to the nature of the projection. One has

$$
h_{i}=\frac{\boldsymbol{X}_{i} \times U}{\left\|\boldsymbol{X}_{i} \times U\right\|}=\frac{x_{i} \times u_{i}}{\left\|x_{i} \times u_{i}\right\|}
$$

where $x_{i}$ denotes the image of any point on the image of the line. In particular (23) provides a mechanism to compute the $h_{i}$ coordinates from the image sequence. Since the observed lines are parallel, it follows that $U$ is orthogonal to the set of the coordinates $h_{i}, i=1 \ldots n$ and therefore, the measurements of the runway axis $U$ can be specified up to a sign that should be fixed by the operator

$$
U= \pm \frac{h_{i} \times h_{j}}{\left\|h_{i} \times h_{j}\right\|} \quad i \neq j .
$$

The construction discussed above is shown in Fig. 5. In the sequel only the measurable variables $\left(h_{i}, U\right)$ are used to design the image-based controller.

The image features for the glide phase are derived from the coordinates of some specific marks on the runway and the runway axis (see Fig. 4). For instance, we propose to use the front corners of the runway along with a prespecified desired descent direction. More precisely the 


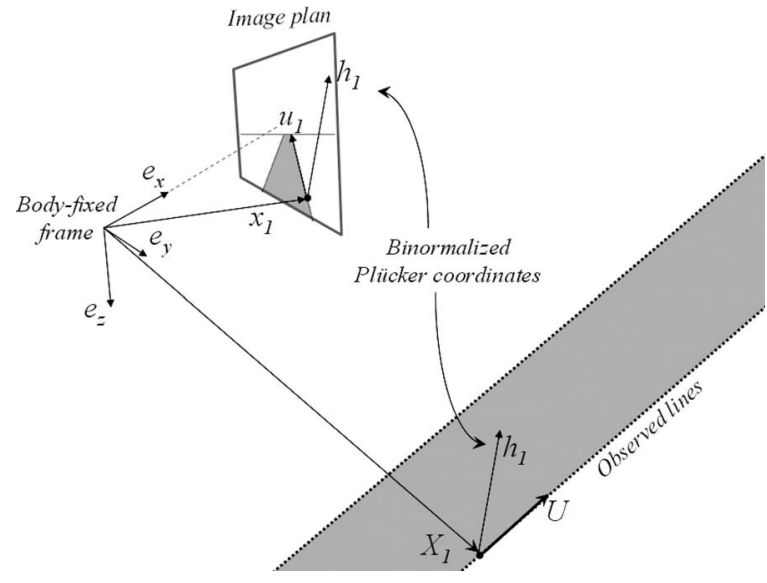

Fig. 5. Binormalized Plücker coordinate $h_{1}$ of a line.

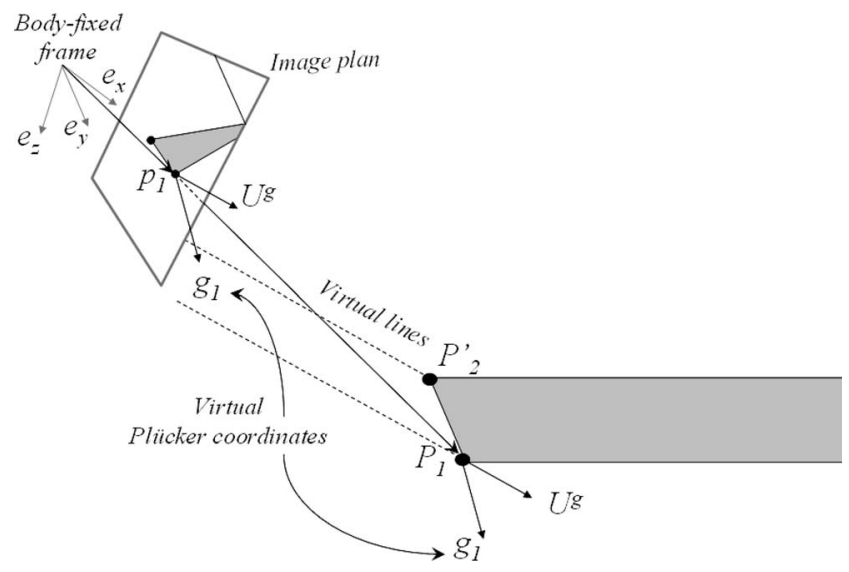

Fig. 6. Binormalized Plücker coordinates of the virtual line used in glide control of the vehicle.

image features used for the glide phase of landing are the Plücker-like coordinates of the virtual line derived from the image coordinates of the first corners of the runway and the desired glide direction. The corners positions are denoted $P_{i}^{\prime} \in I$ in the inertial frame. The virtual lines are derived from a prespecified glide direction $U^{g} \in \mathcal{B}$ defined in the body-fixed frame. Given that the direction of the inertial vertical $E_{z}$ is not collinear to $U$ (the runway is not vertical), it is natural to choose $U^{g}$ in the plane spanned by $E_{z}$ and $U\left(U^{g} \in \operatorname{span}\left\{E_{z}, U\right\}\right)$. One can write

$$
U^{g}=\frac{\cos \gamma^{\star} U+\sin \gamma^{\star} E_{z}}{\left|\cos \gamma^{\star} U+\sin \gamma^{\star} E_{z}\right|}
$$

where $\gamma^{\star}$ is the angle of the desired glide slope with respect to the ground. Since $\gamma^{\star}$ is constant along the glide path, it is straightforward to verify that the inertial direction $u^{g}=R U^{g}$ is also constant.

The points $P_{i}^{\prime}$ can be written as $P_{i}:=R^{\top}\left(\xi-P_{i}^{\prime}\right)$ in the body-fixed frame. One has

$$
g_{i}=\frac{P_{i} \times U^{g}}{\left|P_{i} \times U^{g}\right|}=\frac{p_{i} \times U^{g}}{\left|p_{i} \times U^{g}\right|}
$$

where $p_{i}$ denotes the image of the $i$-th corner of the runway. Fig. 6 describes the notation introduced for these new coordinates.
Recalling that $u(u=R U)$ and $u^{g}\left(u^{g}=R U^{g}\right)$ are constant directions in the inertial frame, it follows that

$$
\dot{U}=-\Omega \times U \text { and } \dot{U}^{g}=-\Omega \times U^{g} .
$$

Given that $X_{i}$ (respectively $P_{i}$ ) is the vector of a fixed point in the inertial frame expressed in the body-fixed frame, one obtains

$$
\dot{X}_{i}=-\Omega \times X_{i}-v \text { and } \dot{P}_{i}=-\Omega \times P_{i}-v .
$$

Using the expression of (23) (respectively (25)), it follows

$$
\begin{gathered}
\dot{h}_{i}=-\Omega \times h_{i}-\frac{1}{r\left(X_{i}, U\right)} \pi_{h_{i}} v \times U \\
\dot{g}_{i}=-\Omega \times g_{i}-\frac{1}{r\left(P_{i}, U^{g}\right)} \pi_{g_{i}} v \times U^{g}
\end{gathered}
$$

where $r\left(X_{i}, U\right)=\left|X_{i} \times U\right|\left(\right.$ respectively $\left.r\left(P_{i}, U^{g}\right)\right)$ are unknown terms since only the projection $x_{i}$ (respectively $p_{i}$ ) of the points $X_{i}$ (respectively $P_{i}$ ) is derived from the image sequence.

The information from each feature (23) (respectively (25)) is summed together to provide a single centroid-like image feature [10]. Define

$$
q= \begin{cases}q_{h}=\sum_{i=1}^{n} h_{i}, & \text { for alignment } \\ q_{g}=\sum_{i=1}^{n} g_{i}, & \text { for glide approach. }\end{cases}
$$

Without any loss of generality, properties and results are presented in the next section for the alignment maneuver $q=q_{h}$. They can be directly applied to the case where $q=q_{g}$.

\section{A. Control Design}

In this section an adaptive visual servo controller is proposed for the alignment (respectively glide-approach) maneuver. In particular we consider a given centroid $q$ (i.e., $q_{h}$ or $q_{g}$ ), the measurement of $U$ (respectively $U_{g}$ ) along with the measurements of the airspeed magnitude $V_{a}$, the angle-of-attack $\alpha$, the side-slip angle $\beta$, and the gravitational direction, and design the control law by assigning $F_{c}^{\star}$. In order to ensure asymptotic stability of the closed-loop system it is also necessary to estimate the unknown wind velocity on-line.

Let us define $q^{\star}$ as the desired reference of the centroid vector $q$ (28). This reference vector is chosen constant in the inertial frame so that one can exploit the passivity property introduced in [18], [19]. This leads to

$$
\dot{q}^{\star}=-\Omega \times q^{\star} .
$$

Define $\delta_{1}$ to be the error term between $q$ and $q^{*}$ :

$$
\delta_{1}=q-q^{\star} .
$$

Differentiating $q$ (28) and recalling (26) it follows [10] that

$$
\dot{q}=-\Omega \times q-Q(v \times U)
$$

where $Q$ is the Jacobian matrix defined as

$$
Q=\sum \frac{1}{r\left(X_{i}, U\right)}\left(I_{3}-h_{i} h_{i}^{\top}\right) .
$$


This matrix is strictly positive $(Q>0)$. Given that the term $r\left(X_{i}, U\right)$ is unknown it follows that $Q$ is also unknown.

Consider the following assumption.

ASSUMPTION 1: For each stage of the control task considered (alignment and glide) there exist two strictly positive scalars $\left(B_{m}, B_{M}\right)>0$ and an open bounded set $D$ that contains the set point for that stage of the control task, such that

$$
\text { for all } \xi \in D, \quad B_{m} I_{3}<Q(\xi)<B_{M} I_{3} .
$$

Note that the basin of attraction of the closed-loop control scheme will lie wholly within in the associated domain $D$ for a given stage of the control task. However, the domains $D$ for the different stages of the control will not be the same, although they will intersect in the zone where switching of the control from one phase to the next is undertaken. A detailed analysis of the nature of the domains $D$ is given in [10].

Thus the dynamics of $\delta_{1}$ are given by

$$
\dot{\delta}_{1}=-\Omega \times \delta_{1}-Q\left[\left(v_{a}+v_{w}\right) \times U\right] .
$$

The controller is designed using the backstepping process in two steps [20].

First step: The air velocity $v_{a}$ is chosen as virtual control input. Since the wind velocity is unknown, it has to be estimated. One of the key contributions of this paper is the design of an estimator $\hat{v}_{w} \in \boldsymbol{B}$ of the crosswind $v_{w} \in \boldsymbol{B}$ that guarantees that

$$
\left|\hat{v}_{w}\right|<\epsilon^{\prime} V_{a}, \forall \epsilon^{\prime} \in\left(\frac{1+\epsilon}{2}, 1\right)
$$

for all time and ensures that $\hat{v}_{w}$ converges to $v_{w}$. Define

$$
\dot{\hat{v}}_{w}=-\Omega_{\times} \hat{v}_{w}+\mathbf{P}_{\hat{v}_{w}} \pi_{U} F_{w}, \quad \hat{v}_{w}(0)=0
$$

where $F_{w}$ is the estimator innovation and

$$
\mathbf{P}_{\hat{v}_{w}}=\epsilon^{\prime} V_{a} \sqrt{1-\frac{\left\|\hat{v}_{w}\right\|^{2}}{\epsilon^{\prime 2} V_{a}^{2}}}\left(I_{3}-\frac{\hat{v}_{w} \hat{v}_{w}^{\top}}{\epsilon^{\prime 2} V_{a}^{2}}\right)>0
$$

is a positive definite matrix. This is justified by the fact that the estimator dynamics allow one to write

$$
\begin{aligned}
\hat{v}_{w} & =\epsilon^{\prime} V_{a} \frac{y}{\sqrt{1+\|y\|^{2}}}, \text { where } \\
\dot{y} & =-\Omega_{\times} y+\pi_{U} u_{w}, \text { and } y(0)=0
\end{aligned}
$$

and guarantees that $\left|\hat{v}_{w}\right| \leq \epsilon^{\prime} V_{a}$.

Introducing the estimate of wind speed into the dynamics of $\delta_{1}(33)$, it yields

$$
\dot{\delta}_{1}=-\Omega_{\times} \delta_{1}+Q\left[U_{\times} v_{a}-\hat{v}_{w}-\tilde{v}_{w}\right]
$$

where $\tilde{v}_{w}=v_{w} \times U-\hat{v}_{w}$ and $\hat{v}_{w}$ represents the estimate of $v_{w} \times U$, i.e., the estimate of crosswind in the plane orthogonal to $U$.

Define a first storage function $S_{1}$

$$
S_{1}=\left|\delta_{1}\right|^{2}+\frac{2}{k_{1}} \tilde{v}_{w}^{\top} \delta_{1}+\frac{4}{k_{1}^{2}}\left|\tilde{v}_{w}\right|^{2}
$$

where $k_{1}$ is a positive control gain. Taking the time derivative of $S_{1}$ and substituting for (34) and (37), it yields

$$
\begin{aligned}
\dot{S}_{1}= & 2 \delta_{1}^{\top} Q\left[U_{\times} v_{a}-\hat{v}_{w}-\tilde{v}_{w}\right]+\frac{2}{k_{1}} \tilde{v}_{w}^{\top} Q\left[U_{\times} v_{a}-\hat{v}_{w}-\tilde{v}_{w}\right] \\
& +\frac{2}{k_{1}} \delta_{1}^{\top} \mathbf{P}_{\hat{v}_{w}} \pi_{U} F_{w}+\frac{8}{k_{1}^{2}} \tilde{v}_{w}^{\top} \mathbf{P}_{\hat{v}_{w}} \pi_{U} F_{w} .
\end{aligned}
$$

Let $v_{a}^{d}$ denote a desired value for the velocity $v_{a}$, defined by

$$
v_{a}^{d}:=U_{\times}\left(k_{1} \delta_{1}-\hat{v}_{w}\right)+\sqrt{V_{a}^{2}-k_{1} \delta_{1}-\hat{v}_{w}^{2}} U
$$

and is chosen such that the storage function $S_{1}$ is monotically decreasing when $v_{a}=v_{a}^{d}$ and $\tilde{v}_{w}=F_{w}=0$. The first term of $v_{a}^{d}$ ensures the convergence of $\delta_{1}$ to zero, while the second term ensures that the aircraft flight direction is aligned with the runway axis.

Note that

$$
\left\|v_{a}^{d}\right\|^{2}=\left\|U_{\times}\left(k_{1} \delta_{1}-\hat{v}_{w}\right)\right\|^{2}+V_{a}^{2}-\left\|k_{1} \delta_{1}-\hat{v}_{w}\right\|^{2}=V_{a}^{2} .
$$

Recalling expressions of $\delta_{1}(29)$ and $\hat{v}_{w}$ (34), it is straightforward to verify that both $\delta_{1}$ and $\hat{v}_{w}$ belong to the plane orthogonal to $U$ and therefore

$$
\left\|U_{\times}\left(k_{1} \delta_{1}-\hat{v}_{w}\right)\right\|=\left\|k_{1} \delta_{1}-\hat{v}_{w}\right\|
$$

It follows that $\left\|v_{a}^{d}\right\|=V_{a}$.

Since the argument of the square root in the definition of $v_{a}^{d}(40)$ has to be positive, the control gain $k_{1}$ must satisfy the following constraint

$$
k_{1}<\frac{\sqrt{1-\epsilon^{\prime}}}{2 n} V_{a} .
$$

Indeed, given that $\left|\delta_{1}\right|<2 n$ and $\left|\hat{v}_{w}\right|<\epsilon^{\prime} V_{a}$, it is straightforward to show that

$$
\left\|k_{1} \delta_{1}-\hat{v}_{w}\right\|<V_{a}
$$

and $v_{a}^{d}$ is well defined. Finally, we define the innovation $F_{w}$ of the wind velocity estimator (34)

$$
F_{w}:=-k_{2} \delta_{1}, \quad k_{2}>0
$$

where $k_{2}$ is a positive control gain.

According to the backstepping process methodology a second error term is introduced. It is defined as follows

$$
\delta_{2}=v_{a}-v_{a}^{d} .
$$

Introducing (43) and the expression of $v_{a}^{d}$ (40) into the dynamics of $\delta_{1}(37)$, one has

$$
\dot{\delta}_{1}=-\Omega_{\times} \delta_{1}-k_{1} Q \delta_{1}-Q \tilde{v}_{w}+Q U_{\times} \delta_{2} .
$$

Consequently, introducing (34), (42), and (40) in the time derivative of $S_{1}$, it follows that

$$
\begin{aligned}
\dot{S}_{1}= & -2 k_{1}\left(\delta_{1}+\frac{\tilde{v}_{w}}{k_{1}}\right)^{\top} Q\left(\delta_{1}+\frac{\tilde{v}_{w}}{k_{1}}\right) \\
& +\frac{2 k_{2}}{k_{1}}\left(\delta_{1}+\frac{4}{k_{1}} \tilde{v}_{w}\right)^{\top} \mathbf{P}_{\hat{v}_{w}} \delta_{1}+2\left(\delta_{1}+\frac{\tilde{v}_{w}}{k_{1}}\right)^{\top} Q U_{\times} \delta_{2} .
\end{aligned}
$$


Using the Schwarz inequality the cross term of the above equation can be bounded as follows:

$$
\begin{aligned}
\left(\delta_{1}+\frac{4}{k_{1}} \tilde{v}_{w}\right)^{\top} \mathbf{P}_{\hat{v}_{w}} \delta_{1}= & -3 \delta_{1}^{\top} \mathbf{P}_{\hat{v}_{w}} \delta_{1} \\
& +4\left(\delta_{1}+\frac{\tilde{v}_{w}}{k_{1}}\right)^{\top} \mathbf{P}_{\hat{v}_{w}} \delta_{1} \\
& \leq-3 \delta_{1}^{\top} \mathbf{P}_{\hat{v}_{w}} \delta_{1}+2 \delta_{1}^{\top} \mathbf{P}_{\hat{v}_{w}} \delta_{1} \\
& +2\left(\delta_{1}+\frac{\tilde{v}_{w}}{k_{1}}\right)^{\top} \mathbf{P}_{\hat{v}_{w}}\left(\delta_{1}+\frac{\tilde{v}_{w}}{k_{1}}\right) \\
& \leq-\delta_{1}^{\top} \mathbf{P}_{\hat{v}_{w}} \delta_{1} \\
& +2\left(\delta_{1}+\frac{\tilde{v}_{w}}{k_{1}}\right)^{\top} \mathbf{P}_{\hat{v}_{w}}\left(\delta_{1}+\frac{\tilde{v}_{w}}{k_{1}}\right)
\end{aligned}
$$

and therefore

$$
\begin{aligned}
\dot{S}_{1} \leq & -2 k_{1}\left(\delta_{1}+\frac{\tilde{v}_{w}}{k_{1}}\right)^{\top}\left(Q-\frac{4 k_{2}}{k_{1}} \mathbf{P}_{\hat{v}_{w}}\right)\left(\delta_{1}+\frac{\tilde{v}_{w}}{k_{1}}\right) \\
& -\frac{2 k_{2}}{k_{1}} \delta_{1}^{\top} \mathbf{P}_{\hat{v}_{w}} \delta_{1}+2\left(\delta_{1}+\frac{\tilde{v}_{w}}{k_{1}}\right)^{\top} Q U_{\times} \delta_{2} .
\end{aligned}
$$

Note that if $\delta_{2}=0$, it is straightforward to verify that $\dot{S}_{1}$ is upper-bounded by a definite negative expression. This completes the first step of the backstepping process.

Second step: Introduce the following storage function

$$
S_{2}=\frac{\left|\delta_{2}\right|}{2^{2}} .
$$

Differentiating $S_{2}$ along trajectories of the closed-loop system one obtains

$$
\begin{aligned}
\dot{S}_{2}= & -v_{a}^{d T} \pi_{v_{a}} F_{c}(\alpha, \mu) \\
& +\delta_{2}^{\top} \mathbf{M}_{v_{a}^{d}}\left(k_{2} \mathbf{P}_{\hat{v}_{w}} \delta_{1}-k_{1} Q U_{\times} \delta_{2}+k_{1}^{2} Q\left(\delta_{1}+\frac{\tilde{v}_{w}}{k_{1}}\right)\right)
\end{aligned}
$$

where

$$
\mathbf{M}_{v_{a}^{d}}=\left(I_{3}-\frac{U v_{a}^{d T} \pi_{U}}{\left\langle U, v_{a}^{d}\right\rangle}\right) U_{\times}
$$

Recalling (40) and choosing $k_{1}$ to satisfy (41), it follows that the term $\left\langle U, v_{a}^{d}\right\rangle$ is strictly positive and the above expression of $\mathbf{M}_{v_{a}^{d}}$ is well defined.

THEOREM 1. Consider the dynamic system given by (9) along with (40) and the estimator (34) with innovation (42). Suppose that assumption 1 is verified. Define the control input

$$
F_{c}^{\star}\left(\alpha^{\star}, \mu^{\star}\right):=4 k_{3} V_{a}^{2} \frac{\pi_{v_{a}} v_{a}^{d}}{\left\|v_{a}+v_{a}^{d}\right\|^{2}}
$$

and the following storage function

$$
L=S_{1}+\frac{1}{k_{1}} S_{2}, k_{1}>0 .
$$

If there exists an $\epsilon_{a} \in(0,2)$ such that

$$
L(0)<\frac{V_{a}\left(2-\epsilon_{a}\right)}{2 k_{1}}
$$

then, there exists a set of positive control gains $\left(k_{1}, k_{2}, k_{3}\right)$ such that $L$ is a Lyapunov function for the closed-loop guidance dynamics, the closed-loop solutions exist for all time and the control terms $\left(\delta_{1}, \delta_{2}\right)$ and the wind velocity estimation error $\left(\tilde{v}_{w}\right)$ converge exponentially to zero.

REMARK 1: The condition on the initial conditions (49) introduced in the theorem ensures that, as long as $\dot{L} \leq 0, v_{a}$ and $v_{d}$ are not in the opposite directions:

$$
1-\cos \left(v_{a}, v_{a}^{d}\right)<2-\epsilon_{a}, \forall t .
$$

PROOF: Consider the first term of $\dot{S}_{2}$ (47) and introduce the control expression (48). Given that $\left\|v_{a}^{d}\right\|=V_{a}$, it follows that

$$
\begin{aligned}
& -4 k_{3} V_{a}^{2} v_{a}^{d T} \pi_{v_{a}} v_{a}^{d} /\left\|v_{a}+v_{a}^{d}\right\|^{2} \\
& =-4 k_{3} V_{a}^{2} v_{a}^{d T}\left(I_{3}-\frac{v_{a} v_{a}^{\top}}{V_{a}^{2}}\right) v_{a}^{d} /\left\|v_{a}+v_{a}^{d}\right\|^{2} \\
& =-4 k_{3} V_{a}^{4}-\left\langle v_{a}^{d}, v_{a}\right\rangle^{2} /\left\|v_{a}+v_{a}^{d}\right\|^{2} \\
& =-4 k_{3}\left(V_{a}^{2}-\left\langle v_{a}^{d}, v_{a}\right\rangle\right)\left(V_{a}^{2}+\left\langle v_{a}^{d}, v_{a}\right\rangle\right) /\left\|v_{a}+v_{a}^{d}\right\|^{2} .
\end{aligned}
$$

Using the fact that $V_{a}^{2}=\left(\left\|v_{a}\right\|^{2}+\left\|v_{a}^{d}\right\|^{2}\right) / 2$, the previous expression is also equal to

$$
\begin{gathered}
-k_{3} \frac{\left(\left\|v_{a}\right\|^{2}-2\left\langle v_{a}^{d}, v_{a}\right\rangle+\left\|v_{a}^{d}\right\|^{2}\right)\left(\left\|v_{a}\right\|^{2}+2\left\langle v_{a}^{d}, v_{a}\right\rangle+\left\|v_{a}^{d}\right\|^{2}\right)}{\left\|v_{a}+v_{a}^{d}\right\|^{2}} \\
=-k_{3} \frac{\left\|v_{a}-v_{a}^{d}\right\|^{2}\left\|v_{a}+v_{a}^{d}\right\|^{2}}{\left\|v_{a}+v_{a}^{d}\right\|^{2}}=-k_{3} \delta_{2} .
\end{gathered}
$$

Using the above relationship and recalling derivative of the first storage function (45), the derivative of $L$ can be bounded (using Schwartz inequality to simplify cross terms) as follows

$$
\begin{aligned}
\dot{L} \leq & -\left(\delta_{1}+\frac{\tilde{v}_{w}}{k_{1}}\right)^{\top}\left(\frac{k_{1}}{2} Q-\frac{4 k_{2}}{k_{1}} \mathbf{P}_{\hat{v}_{w}}\right)\left(\delta_{1}+\frac{\tilde{v}_{w}}{k_{1}}\right) \\
& -\frac{3 k_{2}}{2 k_{1}} \delta_{1}^{\top} \mathbf{P}_{\hat{v}_{w}} \delta_{1}-\frac{k_{3}}{k_{1}}\left\|\delta_{2}\right\|^{2} \\
& +\frac{1}{k_{1}} \delta_{2}^{\top}\left(U_{\times}^{\top} Q U_{\times}+\frac{k_{2}}{2} \mathbf{M}_{v_{a}^{d}} \mathbf{P}_{\hat{v}_{w}} \mathbf{M}_{v_{a}^{d}}^{\top}\right. \\
& \left.-k_{1} \mathbf{M}_{v_{a}^{d}} Q\left(U_{\times}-\frac{k_{1}}{2} \mathbf{M}_{v_{a}^{d}}^{\top}\right)\right) \delta_{2} .
\end{aligned}
$$

To show that the last term of this inequality is negative, it is straightforward to verify that

$$
\left\|\mathbf{M}_{v_{a}^{d}}\right\| \leq\left\|\mathbf{M}_{v_{a}^{d}}\right\|_{\infty}=1+\frac{V_{a}}{\left(1-\epsilon^{\prime}\right) V_{a}-2 n k_{1}} .
$$

Recalling assumption 1, using the fact that $\mathbf{P}_{\hat{v}_{w}}$ is positive definite, and choosing control gains to satisfy

$$
\begin{aligned}
& k_{1}<\frac{\sqrt{1-\epsilon^{\prime 2}}}{2 n} V_{a} \\
& k_{2}<\frac{B_{m} k_{1}^{2}}{8 \epsilon^{\prime} V_{a}} \\
& k_{3}>B_{M}+B_{M} k_{1}^{2}\left\|\mathbf{M}_{v_{a}^{d}}\right\|_{\infty}+\frac{k_{1}^{2} B_{M}+k_{2} \epsilon^{\prime} V_{a}}{2}\left\|\mathbf{M}_{v_{a}^{d}}\right\|_{\infty}^{2}
\end{aligned}
$$


it follows that $\dot{L}$ is bounded by a definite negative expression in $\left(\mathbf{P}_{\hat{v}_{w}} \delta_{1}, \delta_{1}-\frac{1}{k_{1}} \tilde{v}_{w}, \delta_{2}\right)$. Since $\mathbf{P}_{\hat{v}_{w}}$ is a positive definite it follows that $\left(\delta_{1}, \tilde{v}_{w}, \delta_{2}\right)$ converge exponentially to zero. This complete the proof.

This result shows that, as long as the magnitude of the wind velocity is smaller than airspeed $V_{a}$ and that the desired air velocity $v_{a}^{d}(40)$ is not in the opposite direction of $v_{a}$, the desired control action $F_{c}^{\star}$ for the guidance problem ensures exponential convergence of the wind estimate to the real one and exponential stability of $q$ to $q^{\star}$ and therefore guarantees that the airplane follows the desired landing path. The stability of the complete system, including the orientation dynamics requires however a specific gain tuning. Since a high gain inner-loop flight control is required, a small gain controller should be employed for the above outer-loop. In practice the trade-off between the gains tuning between the outer-loop and the inner-loop flight control is an outstanding problem. It affects the performance and the stability domain of the control strategy.

\section{SIMULATION STUDY}

In this section the proposed control algorithm is validated using the realistic simulation architecture $A^{3}$, developed within the LRBA. ${ }^{2}$ This software models an aircraft with an onboard camera. It is based on FightGear software to generate synthetic images that resemble what would be provided by an onboard camera and integrates the aircraft and the onboard camera models. The simulations are undertaken along with an image rate of $100 \mathrm{~Hz}$ and by generating $500 \times 500$ pixels images.

The image processing considered consists of a $2 \mathrm{D}$ tracking procedure for the runway using an active contour approach called corner tracker snake [21]. It is particularly effective for detecting edges and the threshold ends of the runway. The approach can also detect multiple runways in parallel. A detection step is used to initialize the tracking based on visual information (in particular, key points). During the tracking the algorithm estimates the quality of the tracking based on a difference between the reference and the actual object tracked. The algorithm is robust to differing weather conditions including rain or snow as long as there is a sufficient contrast between the runway and the surrounding terrain. More details on the image processing algorithm used or robustness of the detection procedure with respect to weather conditions can be found in [22].

The aircraft considered is a business jet of 16 tons and $20 \mathrm{~m}$ wingspan, whose speed is regulated to $80 \mathrm{~ms}^{-1}$. The runway considered is $1.5 \mathrm{~km}$ long and $40 \mathrm{~m}$ wide. The glide path chosen is a straight slope centered on the runway and passing $50 \mathrm{~m}$ above the initial threshold of the runway. The maneuvers simulated are the following.

\footnotetext{
${ }^{2}$ Laboratoire de Recherches Balistiques et Aérodynamiques of the French Ministry of Defense.
}

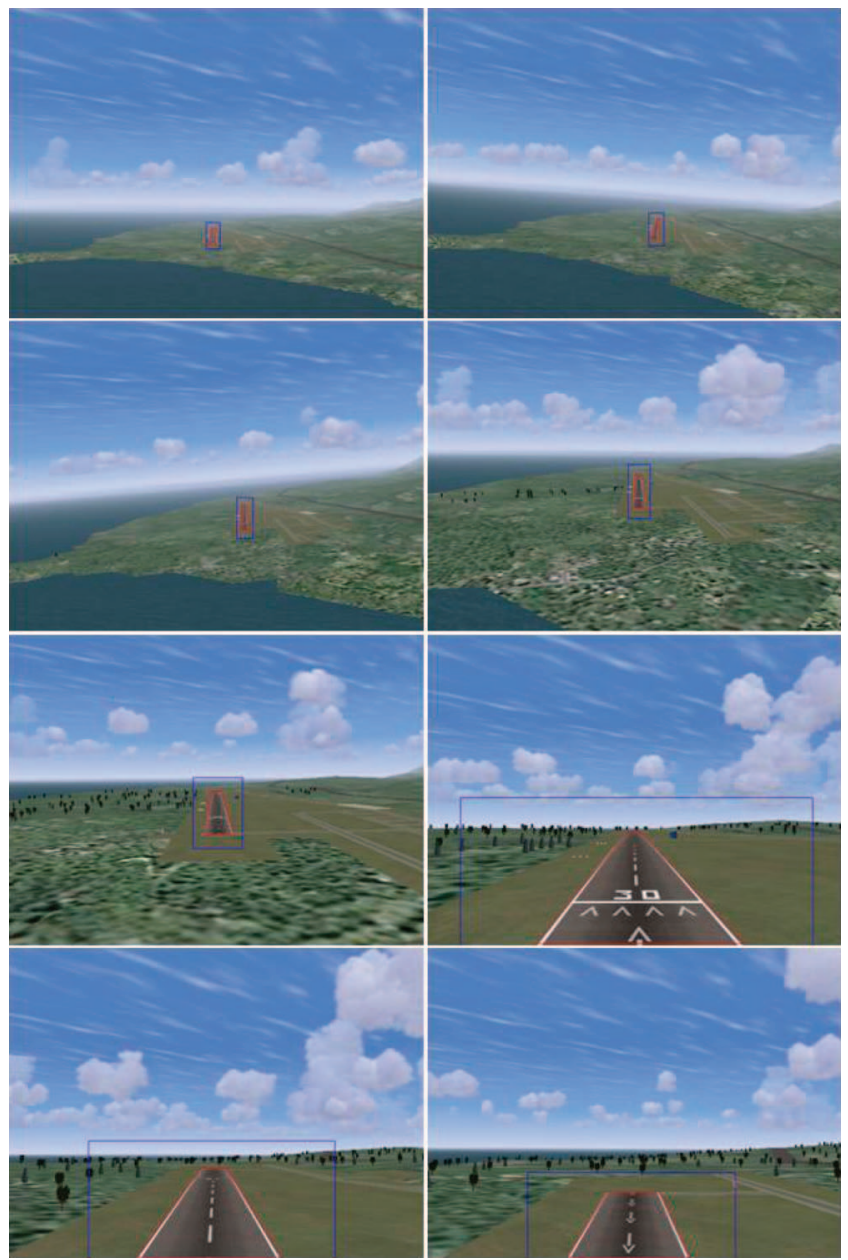

Fig. 7. Images taken from a sequence captured during a landing simulated with $A_{3}$ simulation architecture. The red polygons result from the detection of the runway by the image processing algorithm [21]. The

blue rectangle is a research area initialized at the beginning of the tracking and updated on-line. Images are taken at times $\{0,5,10,20,30$, $40,50,60\}$ seconds.

1) The aircraft realizes an alignment maneuver until its path intersects the slope of the glidepath.

2) The aircraft is stabilized on the glide-slope, including alignment if this was not completed before reaching the glide slope plane.

3) When the vehicle reaches the runway threshold it is stabilized to fly along the runway at constant height, in preparation for a flare manoeuvre.

The actual flare manoeuvre is not undertaken in this simulation and the vehicle will continue along the runway as though aborting the landing. The performance of the proposed control algorithm depends fundamentally on the reliability of the information derived from the image sequence. In the simulations undertaken, the $A^{3}$ simulator has constraints on the maximum image resolution that can be derived from the image rendering software. In order that the edges of the runway are remaining visible throughout the glide phase and during the second alignment phase of landing manoeuvre, a relatively large 

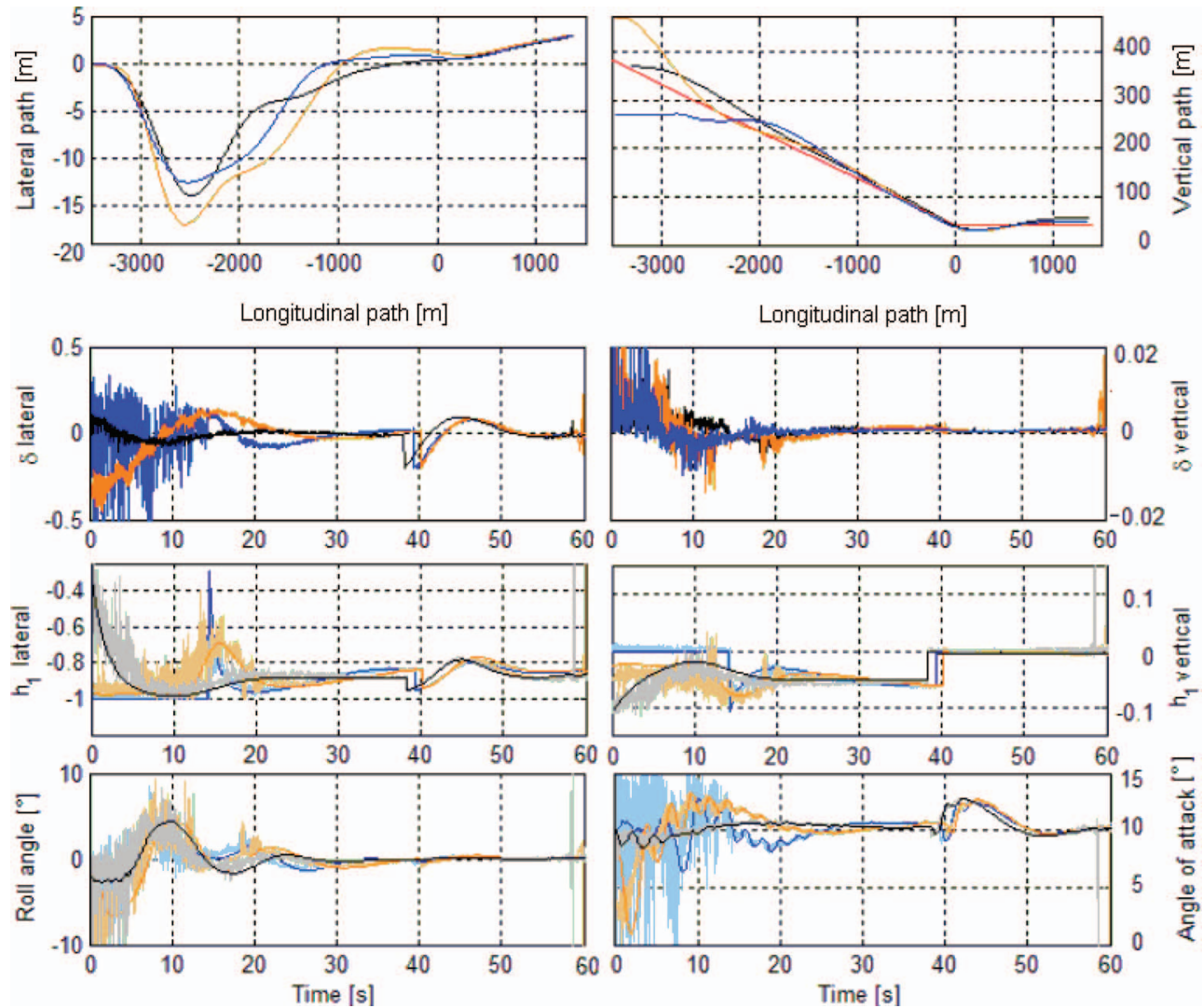

Fig. 8. Simulation results of the landing for different initial heights.

field of view for the camera was required. The result of these constraints is that there is a maximum distance of the simulated aircraft from the runway, and relative perspective of the runway, that yield an image sequence, generated by the simulation software, from which the required visual cues can be extracted. For the realistic scenario considered, the runway can be effectively detected and followed for an initial distance up to $3500 \mathrm{~m}$ at $300 \mathrm{~m}$ to $400 \mathrm{~m}$ height. A glide angle of $6 \mathrm{deg}$ was chosen to provide a well-conditioned simulation study with visible dynamic response in the image sequence. ${ }^{3}$ The final height with respect to the runway was chosen to be $50 \mathrm{~m}$ above the ground, a height from which the alignment phase is triggered.

The large variation in magnification of the image during the landing manoeuvre is a fundamental aspect of the task considered. In practice the difficulty could be addressed in a number of ways: firstly by using a very high resolution camera and high quality optics with a wide field of view; secondly by using a mechanical zoom mechanism to widen the field of view of the camera during the glide phase of the landing; or thirdly, and mostly simply, by using two cameras and switching from a

\footnotetext{
${ }^{3}$ Note that 6 deg slope for the glide path is a higher value than the 3 or 4 deg that is usual for an airplane of this type. With a suitable imaging system any desired glide path angle can be easily servo controlled.
}

camera with high magnification to one with a wide field of view when appropriate. As long as suitable calibration of the cameras is undertaken so that the visual zoom mechanism does not change the visual information significantly, the control algorithms proposed in the paper remain well defined in all such cases.

Various simulations were performed with different initial positions and different crosswind conditions. Fig. 7, shows the evolution of images along a full maneuver (without crosswind). For this simulation there was an initial lateral error of $50 \mathrm{~m}$ relatively to the desired glide path. This explains the variation shown in the horizon due to the roll angle variation on the first images of the sequence. At the end of the scenario considered, it is shown that the image of the runway is centered in the image plane, which implies that the aircraft is properly aligned with the runway.

The evolution of the states and control inputs are given in Figs. 8 and 9. More precisely, the following graphs are provided:

1) trajectories of the aircraft in a plane perpendicular to the runway (lateral and vertical displacement),

2) temporal evolution of the control error $\delta_{1}$ according to the two orthogonal components $U$ (or $U_{D}$ ),

3) bi-normalized Plücker coordinate $h_{1}$ (subsequently used in the controller), drawn by overlaying measurements and actual variables, 

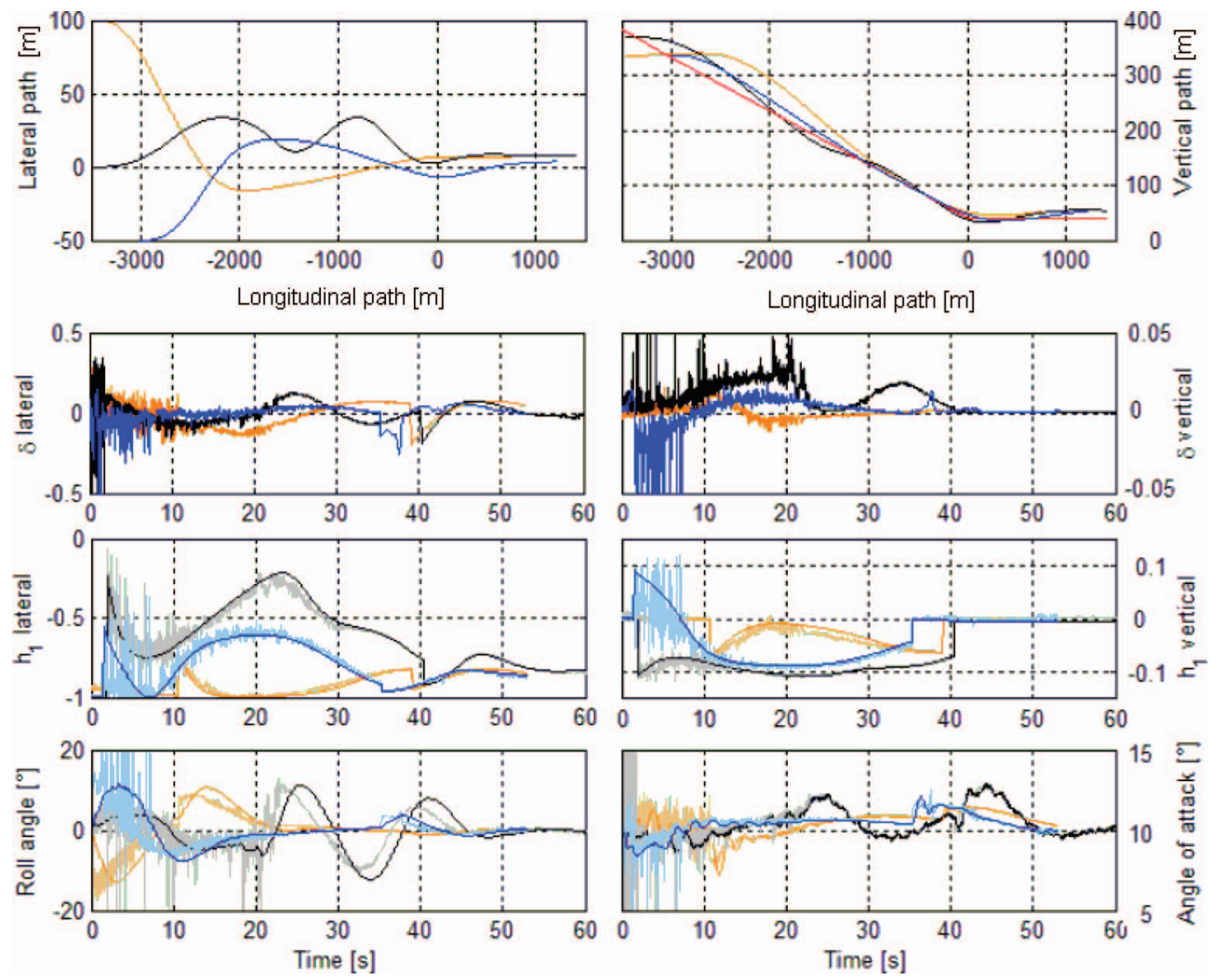

Fig. 9. Simulation results of the landing for different wind conditions and different lateral deviations.

4) guidance control variables in bank angle and angle-of-attack, $\left(\mu^{\star}, \alpha^{\star}\right)$, overlaid with the actual states variables of the airplane $(\mu, \alpha)$.

On Figs. 8 and 9, the variables (the components of the Plücker coordinate $h_{1}$ and the guidance control inputs $\left.\left(\alpha^{\star}, \mu^{\star}\right)\right)$ which are directly affected by image processing disturbances are plotted in lighter colors. It should be noted that the components of Plücker coordinate $h_{1}$ are plotted for the two phases of alignment, whereas it consists of the virtual coordinate $g_{1}$ for the descent phase. This explains the discontinuities in the signals represented.

Fig. 8. shows simulation results performed without crosswind, the colors of graphs refer to the following simulation conditions.

Black: the aircraft is on the desired path (the glide path) at $3500 \mathrm{~m}$ from the runway.

Blue: the aircraft is initially at $100 \mathrm{~m}$ below the desired path, and control starts with a plateau until the slope is reached.

Orange: the aircraft is initially at $100 \mathrm{~m}$ above the desired path, the plane joins directly the desired descent.

Fig. 9 shows the simulation results when significant crosswind magnitude and different lateral errors are introduced. The robustness of the control was tested by introducing significant crosswind and lateral errors.

Black: the aircraft is on the glide path, $3500 \mathrm{~m}$ from the runway with a transverse wind $\left|v_{w}\right|=15 \mathrm{~m} / \mathrm{s}$.
Blue: the aircraft is at $50 \mathrm{~m}$ on the right of the desired descent path, $3000 \mathrm{~m}$ from the runway.

Orange: the aircraft is at $100 \mathrm{~m}$ on the left of the desired descent trajectory, $3500 \mathrm{~m}$ from the runway.

Note that in all results the reference trajectory is followed and initial errors are corrected within a short transient. The effects of the disturbances due to the image processing are negligible even for the glide maneuver despite their high amplitude at high altitude. Moreover the realized trajectories show good transient response with reasonable damping and little or no overshoot. The magnitude of the guidance control inputs along with the effective angle-of-attack and roll angle is small and their variation consists of few degrees with moderate frequency dynamics.

\section{CONCLUSION}

A new image-based visual servo control algorithm has been proposed to perform the alignment and descent maneuvers of an automatic landing. It uses the Plücker coordinates formalism to represent linear features of the runway. The proposed visual servo algorithm is based on the detection of simple visual features within the image of the runway, and requires very little a priori knowledge about the observed environment. The approach can be applied to any type of runway without requiring surveys, differential GPS, or ILS systems. 
The proposed algorithm has been validated using a realistic simulation undertaken in the $A^{3}$ software architecture. The simulations show that the basin of attraction is sufficiently large (initial positioning errors up to $100 \mathrm{~m}$ along with crosswinds until $15 \mathrm{~m} / \mathrm{s}$ ). They also shown that the proposed approach is robust to strong external disturbances due to the realistic image processing and to the extraction of the image features used. The task is performed correctly for initial conditions up to $3.5 \mathrm{~km}$ from the runway, along with lateral errors of $100 \mathrm{~m}$, and unknown crosswinds of up to $15 \mathrm{~ms}^{-1}$. The guidance algorithms have demonstrated a good robustness to initial perturbations and noise measurements and indicate that the control will function effectively in practice.

The final flare to touch down maneuver has not been addressed in this article since the bank-to-turn maneuver is not adapted to such a situation and the image of the runway at a very low height is not compatible with the use of a variant of Plücker coordinates since the runway edges will leave the field of view of the camera. Future work will consider an approach based on optical flow along with a regulation of the roll angle of the aircraft towards zero instead of the bank-to-turn model used in this study.

\section{APPENDIX}

A. Description of the Parameters of (21)

$$
A=\left(\begin{array}{ll}
A_{1} & A_{2}
\end{array}\right)
$$

along with

$$
A_{1}=\left(\begin{array}{ccc}
0 & 0 & 0 \\
\frac{c_{\gamma} s_{\mu}^{\star}}{V_{a}} & \frac{\bar{Q} S C_{z, \alpha}+T_{\gamma}^{\star}}{m V_{a}} & 0 \\
\frac{c_{\gamma} c_{\mu}^{\star}}{V_{a}} & 0 & -\frac{\bar{Q} S C_{y, \beta}+T_{\gamma}^{\star}}{m V_{a}} \\
0 & 0 & 0 \\
0 & \frac{\bar{Q} S l C_{m, \alpha}}{I_{y y}} & 0 \\
0 & 0 & \frac{\bar{Q} S l C_{n, \beta}}{I_{z z}}
\end{array}\right)
$$

[4] Sachs, G. and Moeller, H.
Synthetic vision flight tests for precision approach and landing.

In AIAA Guidance, Navigation and Control Conference, 1995 , pp. 1459-1466.

[5] Chaumette, F. and Hutchinson, S.

Visual servo control, Part I: Basic approaches.

IEEE Robotics and Automation Magazine, 13, 4 (2006), 82-90.

[6] Espiau, B., Chaumette, F., and Rives, P. A new approach to visual servoing in robotics. IEEE Transactions on Robotic and Automation, 8, 3 (1992), 313-326.

[7] Azinheira, J. and Rives, P. Image-based visual servoing for vanishing features and ground lines tracking: Application to a UAV.

and

$$
A_{2}=\left(\begin{array}{ccc}
c_{\alpha^{\star}} & 0 & s_{\alpha^{\star}} \\
0 & 1 & 0 \\
s_{\alpha^{\star}} & 0 & -s_{\alpha^{\star}} \\
\frac{\bar{Q} S l^{2} C_{l, x}}{2 V_{a} I_{x x}} & 0 & \frac{\bar{Q} S l^{2} C_{l, z}}{2 V_{a} I_{x x}} \\
0 & \frac{\bar{Q} S l^{2} C_{m, y}}{2 V_{a} I_{y y}} & 0 \\
0 & 0 & \frac{\bar{Q} S l^{2} C_{n, z}}{2 V_{a} I_{z z}}
\end{array}\right)
$$
International journal of Optomechatronics, 2, 3 (2008), 275-295.

[8] Gonçalves, T., Azinheira, J., and Rives, P. Vision-based automatic approach and landing of fixed-wing aircraft using a dense visual tracking. Informatics in Control Automation and Robotics, Lectures Notes in Electrical Engineering, 85, 3 (2011), 269-282.

[9] Bourquardez, O. and Chaumette, F. Visual servoing airplane for auto-landing. and Systems (IROS'07), 2007, pp. 1314-1319.

[10] Mahony, R. and Hamel, T. Image-based visual servo control of aerial robotic systems using linear image features. IEEE Transactions on Robotics, 21, 2 (2005), 227-239. In IEEE/RSJ InternationalConference on Intelligent Robots 
Classical Mechanics (2nd ed.) (Addison-Wesley Series in Physics). Reading, MA: Addison-Wesley, 1980.

Boiffier, J.-L.

The Flight Dynamics: The Equations. New York: Wiley, 1998, ISBN: 978-0471-942375.

[13] Carter, L. and Shamma, J.

Gain-scheduled bank-to-turn autopilot design using linear parameter varying transformations.

Journal of Guidance, Control, and Dynamics, 19, 5 (1996), 1056-1063.

[14] Cai, G., Duan, G., and Hu, C.

A velocity-based LPV modeling and control framework for airbreathing hypersonic vehicle.

International Journal of Innovative Computing, Information and Control, 7, 5 (2011), 2269-2281.

[15] Marcos, A. and Balas, G.

Development of linear parameter varying models for aircraft. Journal of Guidance, Control and Dynamics, 27 (2004), 218-228.

[16] Serra, P. et al

Nonlinear IBVS controller for the flare maneuver of fixed-wing aircraft using optical flow.

In 49th IEEE Conference on Decision and Control (CDC'2010), 2010, pp. 1656-1661.

[17] Sachs, G., Moeller, H., and Dobler, K. Synthetic vision experiments for approach and taxiing in poor visibility.
In J. Verly (Ed.), Proceedings of SPIE, vol. 2463, Synthetic Vision for Vehicle Guidance and Control, 1995, pp. 128-136.

[18] Hamel, T. and Mahony, R.

Robust visual servoing for under-actuated dynamic systems. In 39th Conference on Decision and Control (CDC'2000), Sydney, Australia, 2000, pp. 3933-3938.

[19] Hamel, T. and Mahony, R.

Visual servoing of an under-actuated dynamic rigid-body system: An image based approach.

IEEE Transactions on Robotics and Automation, 18, 2 (2002), 187-198.

[20] Krstic, M., Kokotovic, P., and Kanellakopoulos, I.

Nonlinear and Adaptive Control Design. NewYork: Wiley, 1995.

[21] Barat, C. and Lagadec, B.

A corner tracker snake approach to segment irregular object shape in video image.

In IEEE International Conference on Acoustics, Speech and Signal Processing (ICASSPJ, 2008), pp. 717-720.

[22] Barat, C. and Phlypo, R.

A fully automated method to detect and segment a manufactured object in an underwater color image. EURASIP Journal on Advances in Signal Processing, 2010 (2010), 1-10.

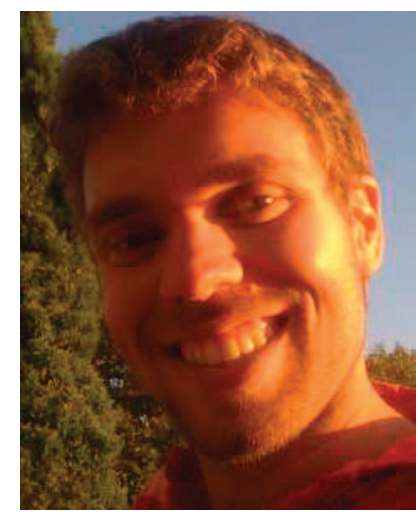

Florent Le Bras received the Engineering Degree in applied mathematics from the Ecole Polytechnique, France, and from the Ecole National Superieure des Techniques Avancees (Paritech) in Sept. 2005, the M.S. degree in automatic and signal processing from Paris-Sud University in 2005, and the Ph.D. in automatic from Nice-Sophia Antipolis University in Dec. 2010.

Parallel to his research activities, he worked as engineer in the Laboratoire de Recherches Balistiques et Aerodynamiques from 2006 to 2010, and in the Technical Directorate of the Direction Generale de l'Armement since June 2010, where he currently holds the rank of Major. 

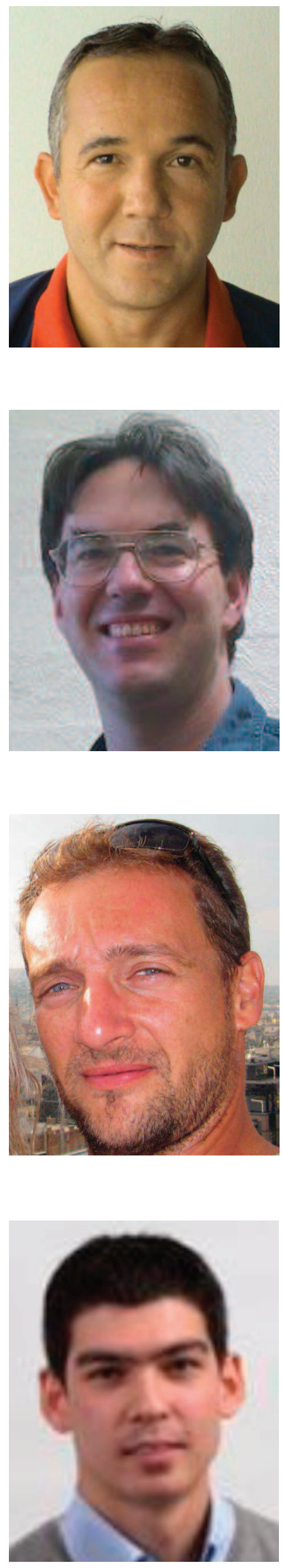

Tarek Hamel received his doctorate degree in robotics from the University of Technologie of Compiègne (UTC), France, in 1996.

After two years as a research assistant at the UTC, he joined the Centre d'Etudes de Mécanique d'lles de France in 1997 as an associate professor. In 2001/2002 he spent one year as CNRS researcher at the Heudiasyc Laboratory. Since 2003 he has been a professor at the University of Nice Sophia Antipolis. His research interests include control theory and robotics with particular focus on nonlinear control, vision-based control and estimation and filtering on Lie groups. He is involved in applications of these techniques to the control of unmanned aerial vehicles and mobile robots.

Dr. Hamel is currently Associate Editor for IEEE Transactions on Robotics and for Control Engineering Practice.

Robert Mahony received a Ph.D. in 1994 (systems engineering) and a BSc in 1989 (applied mathematics and geology) both from the Australian National University.

$\mathrm{He}$ is currently a professor in the Department of Engineering at the Australian National University He worked as a marine seismic geophysicist (1994-1997) and an industrial research scientist before completing a postdoctoral fellowship in France (1997-1999) and a Logan Fellowship at Monash University in Australia (1999-2001). He has held his post at ANU since 2001. His research interests are in nonlinear control theory with applications in mechanical systems and motion systems, mathematical systems theory, and geometric optimization techniques with applications in linear algebra and digital signal processing.

Christian Barat received a Ph.D. in robotics in 1996 from the Université d'Evry Val d'Essonnes.

$\mathrm{He}$ is currently associate professor at the University of Nice Sophia-Antipolis since 2001, and was associate professor at the Université d'Evry Val d'Essonnes from 1997 to 2001. His research interests include pattern recognition, signal processing, intelligent sensor and perception in robotics applications.

Julien Thadasack received the Engineering Degree from the EISTI in 2010. He currently works in DGA Information Superiority in Bruz. 